\title{
On Cooper's and Amici's Double-Star-Measures.
}

I find the following double star observations made by the late $\mathrm{Mr}$. Cooper with the large refractor before it was equatoreally mounted. The aperture was reduced occasionally : -

a urs. min. $1832.32,18^{\prime \prime} .12-6$ Leonis 1833.26, $0 " .79$ and $37^{\prime \prime} .70-.7$ Leonis $1832.32,2^{\prime \prime} .75-7 \mathrm{Vir}-$ ginis 1832.33, 1"94 - o Ophiuchi 1832.32, 3".14 - 4 e Lyrae 1832.31, 3".73 - 5 \& Lyrae 1832.31, $3^{\prime \prime} 10-61$ Cygni 1832.31, 16".82. - It is greatly to be regretted that Amici's measures of the distances between double stars have not been published yet. Narkree. Ireland. N. W. 1877 September 7.
Any measures made so long ago are of importance besides these distances are much smaller than Herschel's. Amici's micrometer was superior to the old wire-micrometers, but I suppose the definition was no better in his telescopes than in Herschel's. I subjoin the only ones I have seen: -

$$
\zeta \text { ursae 1823, 13".27 - } \gamma \text { Virginis 1823.19, 3".30 }
$$

- $\pi$ Boötis 1823, 6".20 - $\zeta$ Boütis 1815..35, 1".00, 1817.j7, 1".100 - — Boötis 181ō.34, 2".31, 1817.57, 2".50 - $\xi$ Boötis 1823.30, 6".67 - $\eta$ Coronae 1817.57, 0".67 — "Herculis 1823, 4".40 - ; Cygni 1823, 33",13.

IV. Doberck.

\section{Elemente des Planeten $\underbrace{163}$.}

Uas nachstehende Elementen-System des am 26. April 1876 von Perrotin entdeckten Planeten wurde aus den vier Orten; April 29, Mai 16 Berlin, Mai 20 Strassburg und Mai 27 Berlin berechnet und stellt die beiden mittleren Beobachtungen - so weit man dies von einer ersten Bahnbestimmung verlangen kann recht befriedigend dar:

Epoche 1876 April $29.0 \mathrm{~m}$. Berl. Z.

L $225^{\circ} 25^{\prime} 57^{\prime \prime}$

II 289.19 .41

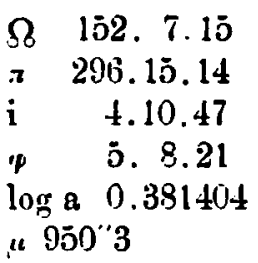

Wien, im September 1877.

\section{Entdeckung eines Cometen.}

Telegraphische Depesche der Kaiserlichen Akademie der Wissenschatten in Wien an den Herausgeber.

Comet Coggia, 13 September $16^{\mathrm{h}} 40^{\mathrm{m}} \mathrm{m}$. Z. Marseille; $"=128^{\circ} 11^{\prime}, \mathrm{P}=41^{\circ} 30^{\circ}$; Bewegung $-11^{\circ}$, $+17^{\prime} ;$ schwach.

Wien 1877 September 15.

\section{Inhalt :}

Zu Nr. 2155. A. Warth. Ephemerides of the five inner Satellites of Saturn. (Schluss). 289. - WV. Dovicik. On Cooper's and Amici's Double-Stars-Measures. 303. - $F . K$. Ginzil. Elemente des Planeten (163). 303. - Entrleckung eines Cometen. Telegraphische Depesche an den Herausgeber. 303 .

Kiel. 1877. September 19. - Druck von C. F. MOHR in Kiel. 